University of East London Institutional Repository: http://roar.uel.ac.uk

This paper is made available online in accordance with publisher policies. Please scroll down to view the document itself. Please refer to the repository record for this item and our policy information available from the repository home page for further information.

To see the final version of this paper please visit the publisher's website.

Access to the published version may require a subscription.

Author(s): Lota, Jaswinder. Al-Janabi, Mohammed., Kale, Izzet

Article Title: Nonlinear-Stability Analysis of Higher Order $\Delta-\Sigma$ Modulators for DC and Sinusoidal Inputs.

Year of publication: 2008

Citation: Lota, J. Al-Janabi, M., Kale, I. (2008) 'Nonlinear-Stability Analysis of Higher Order $\Delta-\Sigma$ Modulators for DC and Sinusoidal Inputs' IEEE Transactions on Instrumentation and Measurement 57 (3) 530-542

Link to published version: (not available)

DOI: (not stated)

Publisher statement:

http://www.ieee.org/web/publications/rights/policies.html 


\title{
Nonlinear-Stability Analysis of Higher Order $\Delta-\Sigma$ Modulators for DC and Sinusoidal Inputs
}

\author{
Jaswinder Lota, Member, IEEE, Mohammed Al-Janabi, Member, IEEE, and Izzet Kale, Member, IEEE
}

\begin{abstract}
The present work that exists on predicting the stability of $\Delta-\Sigma$ modulators is confined to DC input signals and unity quantizer gains. This poses a limitation for numerous $\Delta-\Sigma$ modulator applications. The proposed research work gives the stability curves for DC, sine, and dual sinusoidal inputs for any value of the quantizer gain. The maximum stable input limits for third-, fourth-, and fifth-order Chebyshev-Type-II-based $\Delta-\Sigma$ modulators are established using the describing-function method for DC and sinusoidal inputs. Closed-form mathematical expressions for the gains of the quantizer for higher order $\Delta-\Sigma$ modulators whose inputs are two concurrent sinusoids are derived from first principles. The derived stability curves are shown to agree reasonably well with the simulation results for different types of input signals and amplitudes.
\end{abstract}

Index Terms-DC and sinusoidal inputs, nonlinear, quantizer gain, stability, $\Delta-\Sigma$ modulators.

\section{INTRODUCTION}

$\mathbf{T}$ HE WELL-KNOWN sources of nonlinearity in $\Delta-\Sigma$ modulators are the 1-bit quantizer, op-amp nonlinear DC gain, op-amp slew rate, and nonlinear switch response. The nonlinear op-amp gain and slew rate result in considerable harmonic distortion at the output spectrum of the $\Delta-\Sigma$ modulator. The nonlinear quantizer affects the stability of the $\Delta-\Sigma$ modulator and is therefore the main area of investigation in this paper. The stable input amplitude limits for $\Delta-\Sigma$ modulators are complicated to predict due to the severe nonlinearity of the 1-bit quantizer. To date, various approaches have been applied to more accurately characterize the quantizer [1]-[6], [8], [9]. One technique is to model the quantizer as a threshold function in the state equations. The analysis, however, gets complicated for higher order $\Delta-\Sigma$ modulators and has therefore been limited to the first- and second-order $\Delta-\Sigma$ modulators [1]-[4]. For higher order $\Delta-\Sigma$ modulators, linearized modeling is a method that has been found to be useful for performance analysis [5], [6], [8], wherein the 1-bit quantizer is modeled as a linear gain and an additive noise source. However, apart from performance predictions, the linearized-modeling approach did not previously provide useful stability predictions until a new

Manuscript received January 16, 2007; revised June 14, 2007.

J. Lota and M. Al-Janabi are with the Applied DSP and VLSI Research Group, Department of Electronics, Communications, and Software Engineering, University of Westminster, W1B 2UW London, U.K. (e-mail: jasi@ieee.org; M.Al-Janabi@wmin.ac.uk).

I. Kale is with the Applied DSP and VLSI Research Group, Department of Electronic Systems, University of Westminster, W1B 2UW London, U.K. and also with the Applied DSP and VLSI Research Centre, Eastern Mediterranean University, Gazimagusa, Mersin 10, N. Cyprus (e-mail: kalei@wmin.ac.uk).

Color versions of one or more of the figures in this paper are available online at http://ieeexplore.ieee.org.

Digital Object Identifier 10.1109/TIM.2007.911640

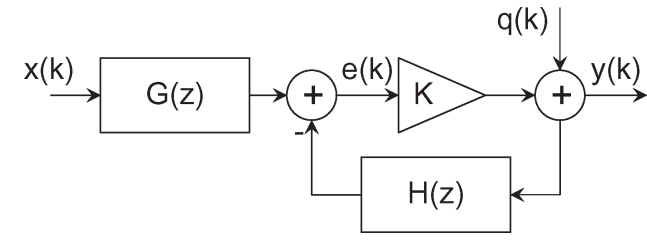

Fig. 1. Quasi-linear $\Delta-\Sigma$ modulator quantizer model.

interpretation of the instability mechanism for $\Delta-\Sigma$ modulators based on the noise-amplification curve was given in [9]. This is however restricted for DC inputs and unity quantizer gains. This quasi-linear method can be extended to more than one input with each input represented by a separate equivalent gain. This concept forms the basis for the describing-function (DF) method [10]. In this paper, the stability analysis based on the noise-amplification curve is accomplished using the DF method for DC single- and dual-tone sinusoidal inputs for nonunity quantizer-gain values. The noise transfer functions (NTFs) of these $\Delta-\Sigma$ modulators utilize Chebyshev-Type-II filters because they achieve better in-band signal-to-noise ratios (SNRs) as compared with Butterworth filters of the same order. In Section II, the quasi-linear stability analysis of $\Delta-\Sigma$ modulators is explained based on the noise-amplification curve. In Section III, the derivation of the noise-amplification curves for DC and sinusoidal inputs with the DF method is given. The simulation results are illustrated and discussed in Section IV followed by the conclusions in Section V.

\section{QUASI-LINEAR-STABILITY ANALYSIS OF $\Delta-\Sigma$ MODULATORS}

A generic $\Delta-\Sigma$ modulator having its quantizer replaced by a gain factor $K$, followed by additive quantization noise $q(k)$ [9], is shown in Fig. 1.

The output of the modulator in the $z$-domain is given by

$$
Y(z)=\operatorname{STF}(z) X(z)+\operatorname{NTF}(z) Q(z)
$$

where $Y(z), X(z)$, and $Q(z)$ are the $\{z\}$-transforms of the output, input, and quantizer noise signals, respectively. The $\operatorname{STF}(z)$ and $\operatorname{NTF}(z)$ represent the signal transfer functions (STFs) and NTFs of the $\Delta-\Sigma$ modulator, which are derived from Fig. 1

$$
\begin{aligned}
\operatorname{STF}(z) & =\frac{K G(z)}{1+K \cdot H(z)} \\
\operatorname{NTF}(z) & =\frac{1}{1+K H(z)}
\end{aligned}
$$




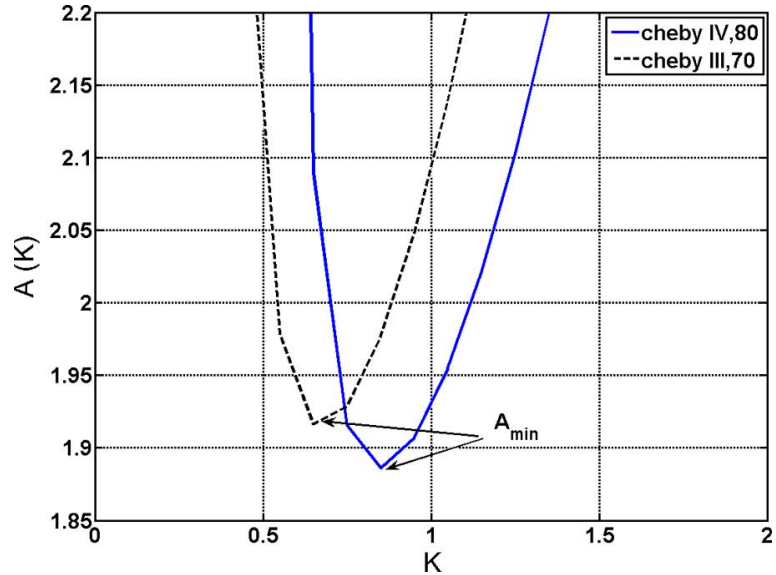

Fig. 2. $A(K)$ curves for some Chebyshev-Type-II NTFs.

It can be seen from (2) and (3) that the poles of the denominator $[1+K H(z)]$ determine the stability of the modulator. For a given loop-filter $H(z)$, there will be a certain interval $\left[K_{\min }, K_{\max }\right]$ for which the modulator is stable [11]. Assuming $q(k)$ to be Gaussian white noise $G\left(0, \sigma_{q}^{2}\right)$ and the transfer function between $q(k)$ and $y(k)$ to be known, then the output noise variance is given by [9]

$$
\operatorname{Var}\{y(k)\}=\sigma_{q}^{2} \int_{0}^{1}\left|\operatorname{NTF}\left(e^{j \pi f}\right)\right|^{2} d f=\sigma_{q}^{2} A(K)
$$

where $\sigma_{q}^{2}$ is the variance of $q(k)$ and $A(K)$ is the total output noise-power-amplification factor. Using Parseval's relation, $A(K)$ can be found in the time-domain as

$$
A(K)=\sum_{k=0}^{\infty}|\mathrm{NTF}(k)|^{2} \triangleq\|\mathrm{NTF}\|_{2}^{2}
$$

where $\operatorname{NTF}(k)$ is the impulse response corresponding to $\operatorname{NTF}(z)$ and $A(K)$ is the squared second-norm of $\operatorname{NTF}(z)$ [9]. The $A(K)$ curves of the loop-filter are crucial for the stability analysis of $\Delta-\Sigma$ modulators. Typical curves for the ChebyshevType-II NTFs are shown in Fig. 2.

The $A_{\min }$ value is the global minimum of the curve. If $K$ increases slightly in the region, where $A(K)$ is monotonically increasing, it results in a higher $A(K)$ value, which leads to more quantization noise transfer into the $\Delta-\Sigma$ modulator. This tends to decrease $K$, leading to a stable equilibrium state [9]. However, where the $A(K)$ curve is monotonically decreasing, even small perturbations can destabilize the modulator. As the signal power increases, the values along the $A(K)$ curve decrease and approach $A_{\min }$. The two values of $K$ come close together and, finally, merge at $A_{\min }$. This characterizes the onset of instability. The modulator-operating region escapes to the left portion of the curve, where it is characterized by low values of $K$. Therefore, for stable operation $A(k)>A_{\min }$ [9]. The $A_{\min }$ values for the Chebyshev-Type-II-based NTFs are shown in Fig. 3.

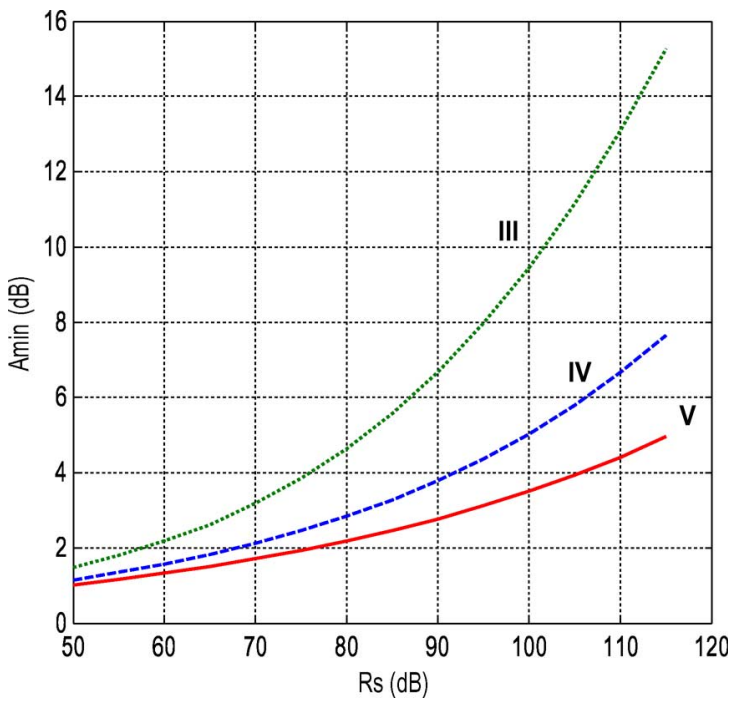

Fig. 3. $A_{\min }$ values versus stop-band attenuation for the third-, fourth-, and fifth-order Chebyshev-Type-II-based NTFs.

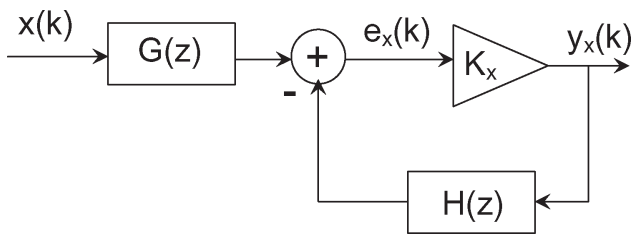

Fig. 4. $\Delta-\Sigma$ modulator linear-signal model.

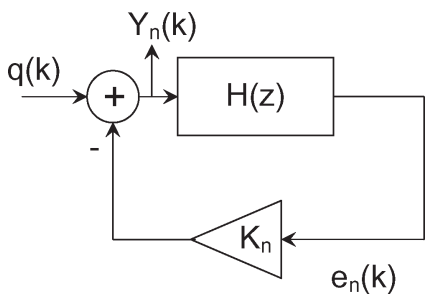

Fig. 5. $\Delta-\Sigma$ modulator linear-noise model.

\section{Noise-Amplification CuRves-DF Method}

Using the DF model, the quantizer-gain $K$ shown in Fig. 1 can be represented with two separate gains $K_{x}$ and $K_{n}$ [6], as shown in Figs. 4 and 5.

Fig. 4 describes the model for the input signal with linear gain $K_{x}$, whereas Fig. 5 describes the noise-signal model with linear gain $K_{n}$. The combined output signal is given by

$$
y(k)=y_{x}(k)+y_{n}(k) .
$$

\section{A. DC Input}

The linearized gains for a 1-bit quantizer with an output $\pm \Delta$ have been calculated in [6] and are given as follows, where $\operatorname{erf}(\cdot)$ is the error function [7]

$$
\begin{aligned}
K_{n} & =2 \frac{\Delta}{\sigma_{e_{n}}^{2}} e^{-m_{e}^{2} / 2 \sigma_{e_{n}}^{2}} \\
K_{x} & =\frac{\Delta}{m_{e}} \operatorname{erf}\left(\frac{m_{e}}{\sigma_{e_{n}} \sqrt{2}}\right)
\end{aligned}
$$


where $m_{e}$ is the mean value of the quantizer input in the signal model, and $\sigma_{e_{n}}^{2}$ is the noise variance input to the quantizer in the noise model. The variance of the output signal is given by

$$
\operatorname{Var}\{y(k)\}=E\left\{y^{2}(k)\right\}-E^{2}\{y(k)\}
$$

where $E\{\cdot\}$ is the expectant operator.

The output signal in the time domain can be expressed as

$$
y(k)=e_{n}(k) K_{n}+q(k)+e_{x}(k) K_{x} .
$$

The first term on the right-hand side of (9) is the power of the output signal, which is given by

$$
\begin{aligned}
& E\left\{y^{2}(k)\right\}=E\left\{e_{n}^{2}(k) K_{n}^{2}\right\}+E\left\{q^{2}(k)\right\}+E\left\{e_{x}^{2}(k) K_{x}^{2}\right\} \\
& E\left\{y^{2}(k)\right\}=\sigma_{e_{n}}^{2} K_{n}^{2}+\sigma_{q}^{2}+m_{e}^{2} K_{x}^{2} .
\end{aligned}
$$

Since the quantization noise is assumed as $G\left(0, \sigma_{q}^{2}\right)$ the mean values of $e_{n}(k)$ and $q(k)$ are equal to zero, then the second term on the right-hand side of (9) becomes

$$
E^{2}\{y(k)\}=m_{e}^{2} K_{x}^{2} .
$$

The resultant variance of the output signal using (9), (12), and (13) becomes

$$
\operatorname{Var}\{y(k)\}=\sigma_{e_{n}}^{2} K_{n}^{2}+\sigma_{q}^{2} .
$$

The noise-power-amplification factor for a DC input signal $A_{\mathrm{dc}}(K)$ after using (4), (7), and (14) simplifies to

$$
A_{\mathrm{dc}}(K)=\frac{\operatorname{Var}\{y(k)\}}{\sigma_{q}^{2}}=\frac{\left(\frac{2}{\pi}\right)\left[e^{-\lambda^{2}}\right]^{2}+\sigma_{q}^{2}}{\sigma_{q}^{2}}
$$

where $\lambda$ is a factor defined as follows: $\lambda=m_{e} / \sigma_{e n} \sqrt{ } 2$, and $\sigma_{q}^{2}$ is the quantization noise power given by [6]

$$
\sigma_{q}^{2}=\Delta^{2}\left[1-\frac{m_{x}}{\Delta^{2}}-\frac{2}{\pi} e^{-2\left[\operatorname{erf}^{-1}\left(\frac{m_{x}}{\Delta}\right)\right]^{2}}\right] .
$$

\section{B. Sinusoidal Input}

The linearized gains for a sinusoidal input and random Gaussian feedback components have been solved for the case of an ideal relay in [12], which can be assumed for a 1-bit quantizer with an output of $\pm \Delta[6]$ and are shown as follows:

$$
\begin{aligned}
K_{n} & =\left(\frac{2}{\pi}\right)^{\frac{1}{2}}\left(\frac{\Delta}{\sigma_{e_{n}}}\right) F\left(\frac{1}{2}, 1,-v^{2}\right) \\
K_{x} & =\left(\frac{2}{\pi}\right)^{\frac{1}{2}}\left(\frac{\Delta}{\sigma_{e_{n}}}\right) F\left(\frac{1}{2}, 2,-v^{2}\right) .
\end{aligned}
$$

Here, $v \underline{\Delta}(a / \sqrt{2})=\left(1 / \sigma_{e_{n}}\right)$, where $a$ is the amplitude of the sinusoidal input signal $x(k)$. The expression $F(\alpha, \gamma, x)$ is the confluent hypergeometric function defined by [13], and $\Gamma$ is a gamma function [7]

$$
F(\alpha, \gamma, \chi) \triangleq 1+\frac{\alpha \chi}{\gamma}+\frac{\alpha(\alpha+1) \chi^{2}}{\gamma(\gamma+1) \Gamma 2}+\cdots
$$

The variance of the output signal is given by

$$
\operatorname{Var}\{y(k)\}=E\left\{y^{2}(k)\right\}-E^{2}\{y(k)\} .
$$

The power of the output signal is given by

$$
\begin{aligned}
& E\left\{y^{2}(k)\right\}=E\left\{e_{n}^{2}(k) K_{n}^{2}\right\}+E\left\{q^{2}(k)\right\}+E\left\{e_{x}^{2}(k) K_{x}^{2}\right\} \\
& E\left\{y^{2}(k)\right\}=\sigma_{e n}^{2} K_{n}^{2}+\sigma_{q_{s}}^{2}+\sigma_{e_{x}}^{2} K_{x}^{2}
\end{aligned}
$$

where $\sigma_{q s}^{2}$ is the quantization noise power for a sinusoidal input. The second term on the right-hand side of (20) is

$$
\begin{aligned}
& E^{2}\{y(k)\}=E^{2}\left\{e_{n}(k) K_{n}\right\}+E^{2}\{q(k)\}+E^{2}\left\{e_{x}(k) K_{x}\right\} \\
& E^{2}\{y(k)\}=E^{2}\left\{e_{x}(k)\right\} K_{x}^{2}
\end{aligned}
$$

where the mean values of $e_{n}(k)$ and $q(k)$ are zero. The input signal is a sinusoid modeled as a random variable (RV) having constant amplitude. Since the phase is random with a uniform probability density function (pdf) $E\left\{e_{x}(k)\right\}=0$. Therefore, from (20) and (24)

$$
\operatorname{Var}\{y(k)\}=E\left\{y^{2}(k)\right\} .
$$

Given that the frequency of $x(k)$ is small in the baseband region, this then results in [6]

$$
\frac{E_{x}(z)}{X(z)} \approx \frac{1}{K_{x}} .
$$

The variance of $e_{x}(k)$ is

$$
\sigma_{e_{x}}^{2}=\frac{1}{K_{x}^{2}} \sigma_{x}^{2}
$$

From (25) and (27), the output-signal variance is

$$
\operatorname{Var}\{y(k)\}=\sigma_{q_{s}}^{2}+K_{n}^{2} \sigma_{e_{n}}^{2}+\sigma_{x}^{2} .
$$

The output-noise variance is therefore

$$
\operatorname{Var}_{n}\{y(k)\}=\sigma_{q_{s}}^{2}+K_{n}^{2} \sigma_{e_{n}}^{2} .
$$

Substituting (17) in (29), the noise-amplification factor for a sinusoidal input signal becomes

$$
A_{\sin e}(K)=\frac{\left(\frac{2}{\pi}\right) F^{2}\left(\frac{1}{2}, 1,-v^{2}\right)+\sigma_{q_{s}}^{2}}{\sigma_{q_{s}}^{2}} .
$$


The values of $v$ and $\sigma_{q s}^{2}$ can be found using the following expressions derived in [6]:

$$
\begin{aligned}
& v^{2} F^{2}\left(\frac{1}{2}, 2,-v^{2}\right)=\frac{\pi}{4}\left(\frac{a^{2}}{\Delta^{2}}\right) \\
& \sigma_{q_{s}}^{2}=\Delta^{2}\left[1-\frac{a^{2}}{2 \Delta^{2}}-\frac{2}{\pi} F^{2}\left(\frac{1}{2}, 1,-v^{2}\right)\right] .
\end{aligned}
$$

\section{Two Sinusoidal Inputs (Incommensurate)}

The linearized gains for two sinusoidal input signals $x_{a}(t)=a \cos \left(w_{1} t+\phi_{1}\right), x_{b}(t)=b \cos \left(w_{2} t+\phi_{2}\right)$ and a random Gaussian signal representing the feedback components have been solved for the case of the 1-bit quantizer, as shown in the Appendix, where the final expressions are given by

$$
K_{a}=\left(\frac{2}{\pi}\right)^{\frac{5}{2}}\left(\frac{\Delta}{\sigma}\right)\left(\frac{b}{a}\right)\left(\frac{1}{\frac{1}{2}-\rho_{b}^{2}}\right)\left\{{ }_{1} F_{1}\left(1, \frac{3}{2},-\rho_{a}^{2}\right)+\psi_{a}\right\}
$$

$$
K_{b}=\left(\frac{2}{\pi}\right)^{\frac{5}{2}}\left(\frac{\Delta}{\sigma}\right)\left(\frac{a}{b}\right)\left(\frac{1}{\frac{1}{2}-\rho_{a}^{2}}\right)\left\{1 F_{1}\left(1, \frac{3}{2},-\rho_{b}^{2}\right)+\psi_{b}\right\}
$$

$K_{n}=\sqrt{\frac{2}{\pi}}\left(\frac{\Delta}{\sigma}\right) e^{-\rho_{a}^{2}} e^{-\rho_{b}^{2}} \zeta$

where

$$
\begin{aligned}
\psi_{a} & =\left\{\frac{4}{3} \rho_{a}^{2}-\frac{16}{45} \rho_{a}^{4}+\frac{16}{175} \rho_{a}^{6}-\frac{128}{6615} \rho_{a}^{8}+\cdots\right\} \\
\psi_{b} & =\left\{\frac{4}{3} \rho_{b}^{2}-\frac{16}{45} \rho_{b}^{4}+\frac{16}{175} \rho_{b}^{6}-\frac{128}{6615} \rho_{b}^{8}+\cdots\right\} \\
\zeta & =\left\{1+\rho_{a}^{2} \rho_{b}^{2}+\frac{\rho_{a}^{4} \rho_{b}^{4}}{4}+\frac{\rho_{a}^{6} \rho_{b}^{6}}{36}+\frac{\rho_{a}^{8} \rho_{b}^{8}}{576}+\cdots\right\}
\end{aligned}
$$

and $\rho_{a}^{2}=(1 / 2)\left(a^{2} / \sigma^{2}\right) ; \rho_{b}^{2}=(1 / 2)\left(b^{2} / \sigma^{2}\right)$.

From (29), the output-noise variance is given by

$$
\operatorname{Var}\{y(k)\}=\sigma_{e_{n}}^{2} K_{n}^{2}+\sigma_{q_{a b}}^{2}
$$

where $\sigma_{q_{a b}}^{2}$ is the quantization noise power for the two uncorrelated sinusoidal inputs $x_{a}(t)$ and $x_{b}(t)$. Therefore, from (35) and (39), the noise-amplification factor is given by

$$
A_{a b}(K)=\frac{\left(\frac{2}{\pi}\right)\left\{e^{-\rho_{a}^{2}} e^{-\rho_{b}^{2}}\right\}^{2} \zeta^{2}+\sigma_{q_{a b}}^{2}}{\sigma_{q_{a b}}^{2}} .
$$

Since $x_{a}(t)$ and $x_{b}(t)$ are uncorrelated, the power of the output signal is given by

$$
E\left\{y^{2}(k)\right\}=\sigma_{e_{n}}^{2} K_{n}^{2}+\sigma_{q_{a b}}^{2}+\sigma_{e_{b}}^{2} K_{b}^{2}+\sigma_{e_{a}}^{2} K_{a}^{2}
$$

where $\sigma_{e_{b}}^{2}$ and $\sigma_{e_{a}}^{2}$ are the powers of the sinusoidal inputs at the quantizer input. From (27), we have

$$
\begin{aligned}
& \sigma_{e_{b}}^{2}=\frac{1}{K_{b}^{2}} \sigma_{b}^{2} \\
& \sigma_{e_{a}}^{2}=\frac{1}{K_{a}^{2}} \sigma_{a}^{2} .
\end{aligned}
$$

From (35), (41), and (42), we get

$$
\Delta^{2}=\frac{2}{\pi} \Delta^{2}\left\{e^{-\rho_{a}^{2}} e^{-\rho_{b}^{2}}\right\}^{2} \zeta^{2}+\sigma_{q_{a b}}^{2}+\frac{b^{2}}{2}+\frac{a^{2}}{2} .
$$

Rearranging (43), the quantization noise power is given by

$$
\sigma_{q_{a b}}^{2}=\Delta^{2}\left[1-\frac{a^{2}}{2 \Delta^{2}}-\frac{b^{2}}{2 \Delta^{2}}-\frac{2}{\pi}\left\{e^{-\rho_{a}^{2}} e^{-\rho_{b}^{2}}\right\}^{2} \zeta^{2}\right] .
$$

From (34) and (42), we get

$$
\left(\frac{2}{\pi}\right)^{5}\left(\frac{a^{2}}{b^{2}}\right) \frac{\rho_{b}^{2}}{\left[\frac{1}{2}-\rho_{a}^{2}\right]^{2}}\left\{{ }_{1} F_{1}\left(1, \frac{3}{2},-\rho_{b}^{2}\right)+\psi_{b}\right\}^{2}=\frac{b^{2}}{2} .
$$

Similarly, from (33) and (42) for the sinusoid $x_{a}(t)$, we have

$$
\left(\frac{2}{\pi}\right)^{5}\left(\frac{b^{2}}{a^{2}}\right) \frac{\rho_{a}^{2}}{\left[\frac{1}{2}-\rho_{b}^{2}\right]^{2}}\left\{{ }_{1} F_{1}\left(1, \frac{3}{2},-\rho_{a}^{2}\right)+\psi_{a}\right\}^{2}=\frac{a^{2}}{2} .
$$

The two simultaneous (45) and (46) were solved by deploying the MATLAB Symbolic Toolbox in order to get the values of $\rho_{a}$ and $\rho_{b}$ for various values of $a$ and $b$.

In Sections I-III, we have seen that the noise-amplification factor can be determined in two ways, viz., statistically and numerically. Statistically, it can be derived from (4), provided that the noise and signal quantizer gains are known. The quantizer gain is therefore split up as signal and noise quantizer gains using the DF method. The derived noise-amplification factor here is a function of the signal amplitude and the quantization noise power. In case the of DC and single-sine inputs, the signal and noise gains have been used from the nonlinear-control theory. Equations (15) and (30) give the statistically derived noise-amplification factor for DC and single-sine inputs. For the dual sinusoidal input, the quantizer gains have been derived from the Appendix. The noise-amplification factor is arrived from (40).

The noise-amplification factor can also be derived numerically from (3) and (5). Here, the parameter is a function of the quantizer gain and the NTF, as shown in Fig. 2. The $A_{\min }$ value is the global minimum value of the curve. To ensure stability, the value of the noise-amplification factor must always exceed $A_{\min }$. Therefore, from the statistically derived noiseamplification factor (which is a function of the input signal and noise power), we can infer the values of the input amplitude, for which its noise-amplification factor is always greater than $A_{\min }$, to ensure the stability of the $\Delta-\Sigma$ modulator for a 


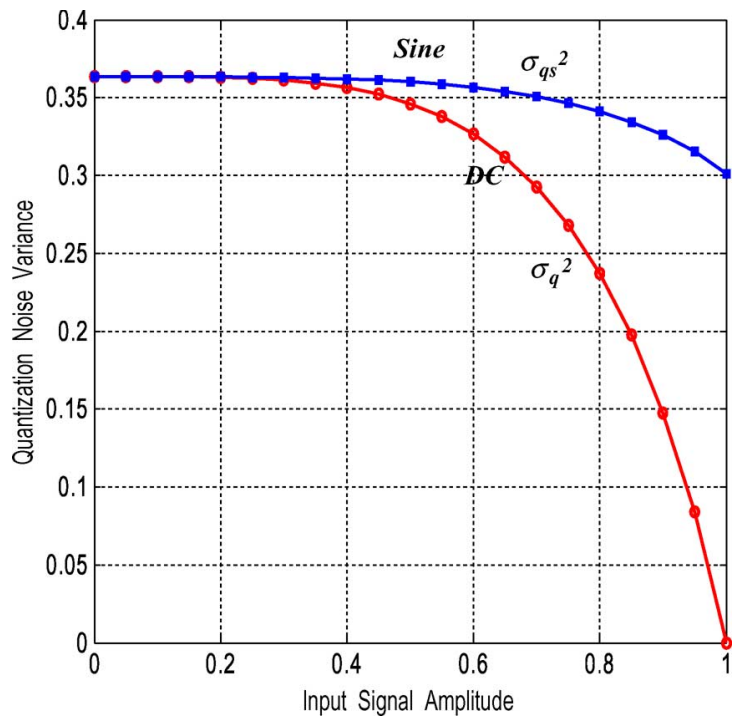

Fig. 6. Quantization noise for DC and sinusoidal inputs.

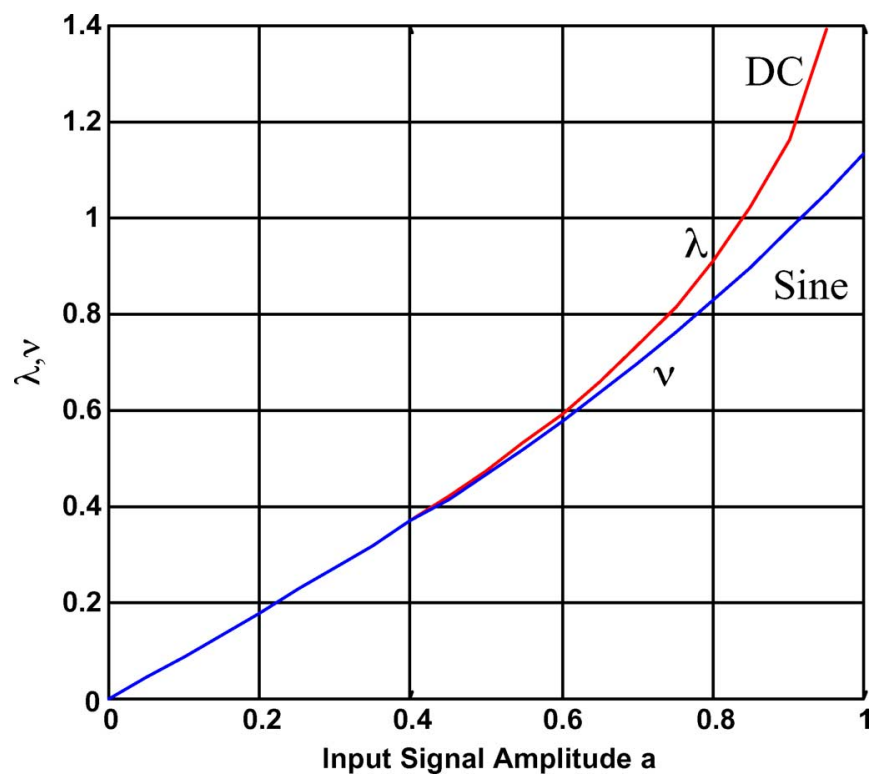

Fig. 7. Variation of $v$ (sine) and $\lambda$ (DC) versus the input-signal amplitude.

particular NTF. The derived stability curves for a given NTF can therefore be plotted and will be covered in the next section.

\section{Results AND Simulations}

\section{A. DC and Single Sinusoidal Inputs}

The variation of the DC and sinusoidal-input quantization noise power $\sigma_{q}^{2}$ and $\sigma_{q s}^{2}$, with respect to the input-signal amplitude using (16) and (32), are shown in Fig. 6.

As shown, $\sigma_{q}^{2}$ decreases and becomes zero as the input-signal amplitude increases to unity. The quantization noise power $\sigma_{q s}^{2}$ does not decrease to zero and remains at 0.3 for an input amplitude of 1.0. Equation (31) has been solved for $v$ up to the tenth power of $v$ using the MATLAB Symbolic Toolbox.

Fig. 7 shows the variation of $\lambda$ and $v$, with respect to the input-signal amplitude.

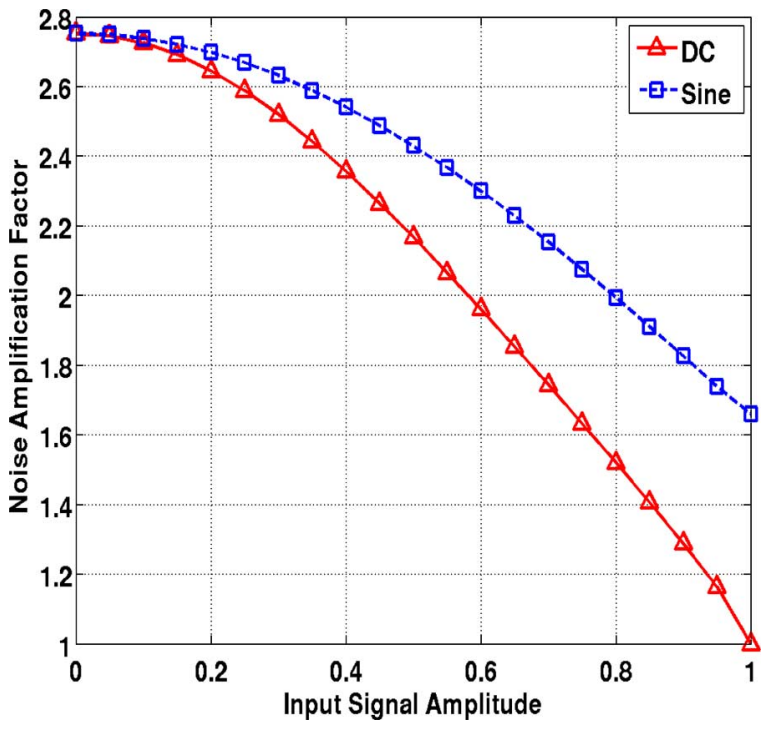

Fig. 8. Noise-amplification factor for sinusoidal and DC inputs.

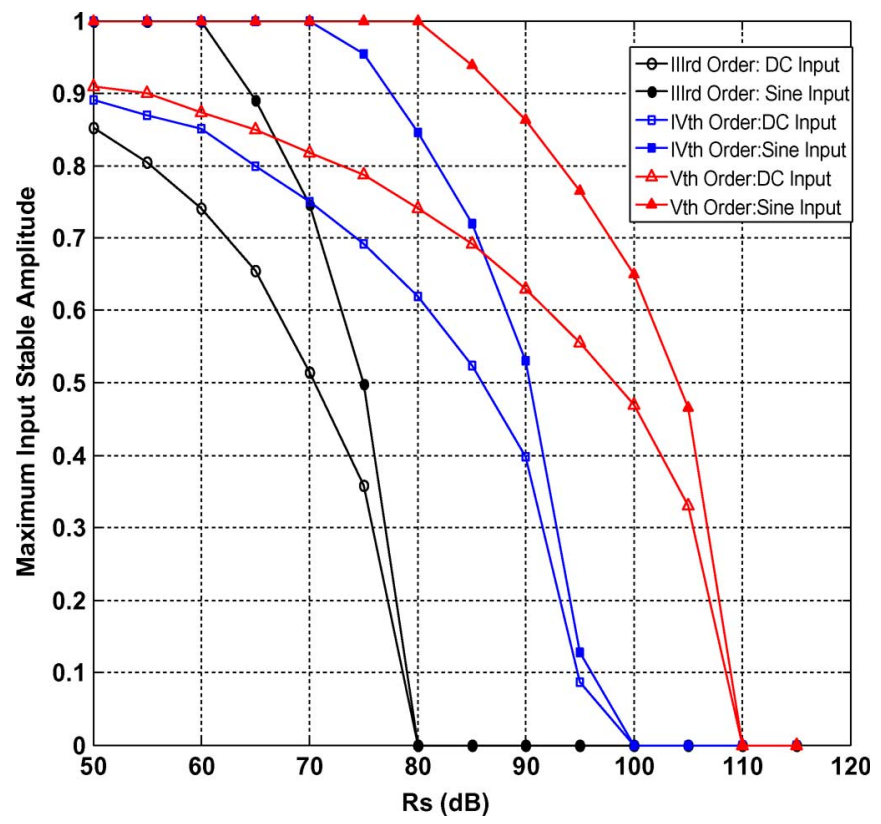

Fig. 9. Stable-input amplitude for Chebyshev-Type-II NTF(z).

It has been observed that, for amplitudes less than 0.4, the quantization noise $\lambda$ and $v$ are almost the same for DC and sinusoidal inputs. This coincides with the fact that, in nonlinear feedback systems, the effective gain of the nonlinearity on a small signal is independent of the signal type [10]. The noiseamplification factors $A_{\mathrm{dc}}(K)$ and $A_{\mathrm{sin}}(K)$ using (15) and (30) are shown in Fig. 8. It is shown that the values of $A_{\mathrm{dc}}(K)$ using the DF method are the same as in [9].

Using $A_{\mathrm{dc}}(K)$ and $A_{\sin }(K)$, the maximum stable input amplitudes for the third-, fourth-, and fifth-order ChebyshevType-II-based $\Delta-\Sigma$ modulator are shown in Fig. 9.

However, these are true for unity values of quantizer gain $K$. The variations of the stable sinusoidal input amplitude for the third-, fourth-, and fifth-order Chebyshev-Type-II-based $\Delta-\Sigma$ modulator in relation to the quantizer gain $K$ and the stop-band 


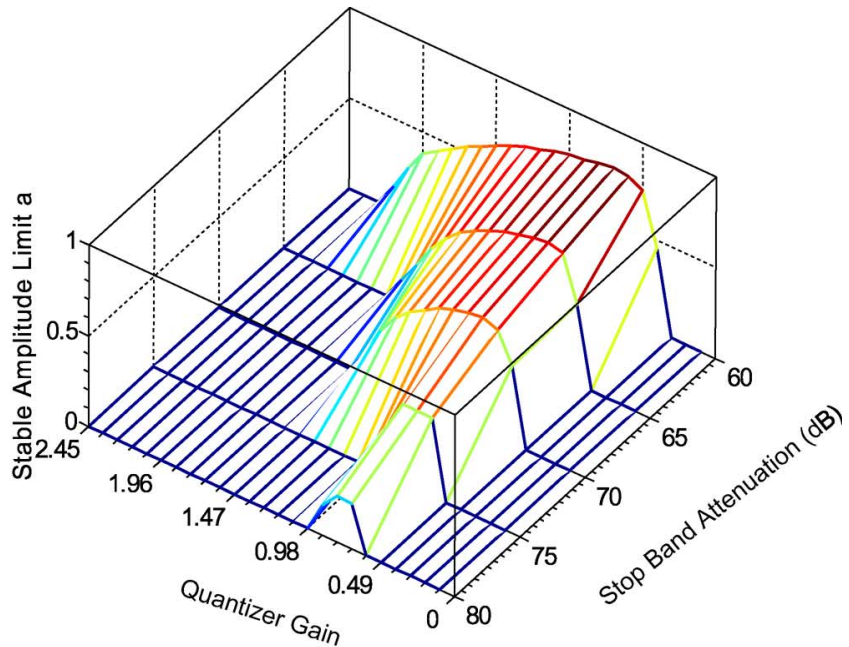

(a)

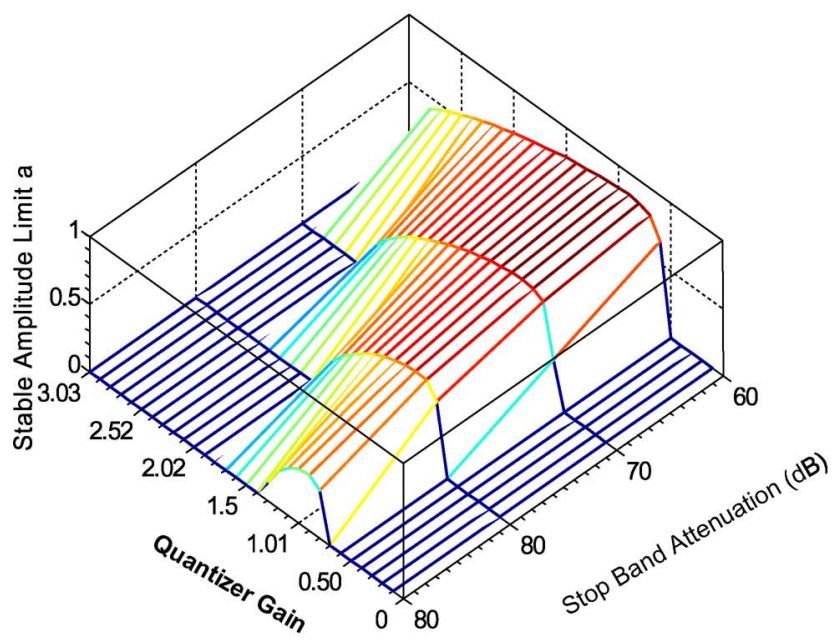

(b)

Fig. 10. (a) Stable amplitude limit for third order for DC input. (b) Stable amplitude limit for fourth order for DC input.

attenuation are shown in Fig. 10(a) and (b) for a DC input and in Fig. 11(a) and (b) for a sinusoidal input, respectively.

For comparison, the stable input-amplitude variation for dc and sinusoidal inputs for a fifth-order Chebyshev-Type-II-based $\Delta-\Sigma$ modulator with a stop-band attenuation of $67 \mathrm{~dB}$ is shown in Fig. 12.

\section{B. Two Sinusoidal Inputs}

From (45) and (46), the values of $\rho_{b}$ have been shown in Fig. 13(a). It is shown that $\rho_{b}$ gets bigger as the amplitude $b$ increases. However, the increase in $\rho_{b}$ gets attenuated as the signal amplitude $a$ increases from 0.2 to 0.8 . As shown, the effect of this attenuation decreases when $b>a$. This becomes more noticeable for $a=0.8$.

The amplitude of $\rho_{a}$, as shown in Fig. 13(b), is seen to gradually decrease as $b$ increases. It is also seen to drop sharply when the amplitude of $b$ becomes greater than $a$.

The values of $\rho_{a}$ and $\rho_{b}$ for the following amplitudes are as follows: $a=0.2,0.4,0.6$, and 0.8 are shown in Fig. 14(a)-(d).

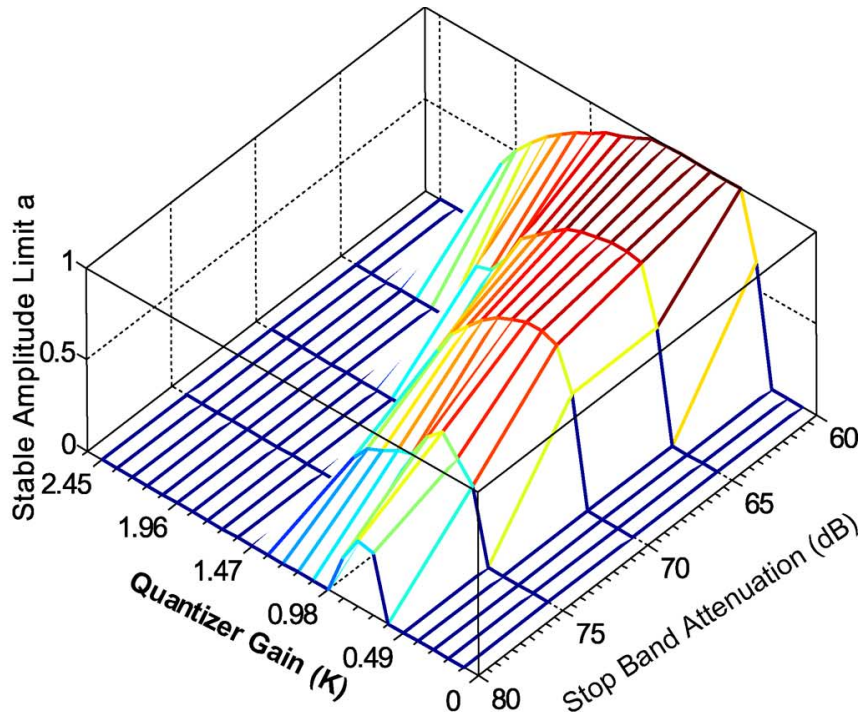

(a)

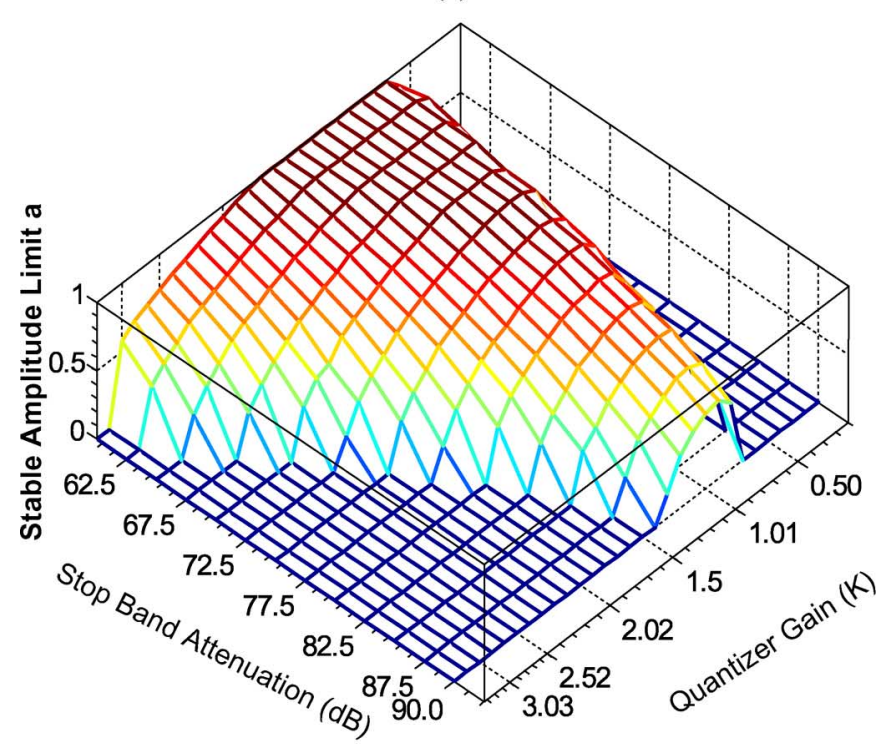

(b)

Fig. 11. (a) Amplitude limit for third order for sinusoidal input. (b) Amplitude limit for fourth order for sinusoidal input.

The magnitudes of $\rho_{a}$ and $\rho_{b}$ become equal when both sinusoids have the same amplitudes, i.e., $a=b$.

Using (44), the quantization noise power $\sigma_{q_{a b}}^{2}$ is plotted in Fig. 15. The $\sigma_{q_{a b}}^{2}$ in the regions $b<0.2, b<0.4$, and $b<0.6$ for the curves A $(a=0.2), \mathrm{B}(a=0.4)$, and C $(a=0.6)$ (left side of the nulls for the three curves), respectively, increases mainly due to $\rho_{a}$. As $\rho_{a}$ becomes bigger when the amplitude $a$ increases from 0.2 to 0.6 in Fig. 13(b), so does $\sigma_{q_{a b}}^{2}$ in this region. The increase in $\sigma_{q_{a b}}^{2}$ in the regions $b>0.2, b>0.4$, and $b>0.6$ (right-hand side of the three nulls) for the curves A, B, and $\mathrm{C}$, respectively, is mainly attributed to $\rho_{b}$. As $\rho_{b}$ increases with a reduction in the amplitude $a$ from 0.6 to 0.2 in Fig. 13(a), so does $\sigma_{q_{a b}}^{2}$.

Since the quantization noise power $\sigma_{q_{a b}}^{2}, \rho_{a}$ and $\rho_{b}$ are known; the same are substituted in (40) to obtain the noiseamplification curves $A_{a b}(K)$ for $a=0.2,0.4$, and 0.6 . These noise-amplification curves $A_{a b}(K)$ are plotted in Fig. 16. 


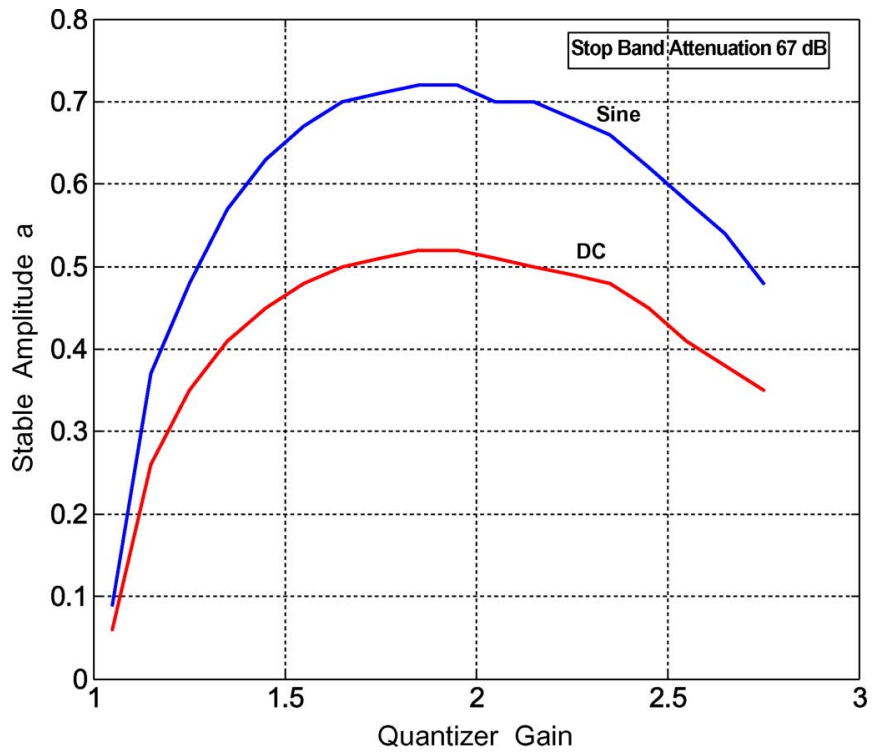

Fig. 12. Stable amplitude variation for DC and sinusoid inputs.

Using the values obtained for $A_{a b}(K)$, the stable amplitude limits for $b$ have been plotted for the third-, fourth-, and fifth-order Chebyshev-Type-II-based NTF for $a=0.2$ and 0.4 in Figs. 17(a)-(c) and 18(a)-(c), respectively.

Simulations for the fifth-order Chebyshev-Type-II-based $\Delta-\Sigma$ modulator, as shown in Fig. 19, were performed for 1400 samples, where the input amplitude was increased in steps of 0.1 . The maximum stable amplitude limits were obtained and compared with simulations as shown in Fig. 20.

The difference between the theoretical and simulated input stability limits is attributed to the presence of more spectral tones when the input to the $\Delta-\Sigma$ modulator is a DC signal. This discrepancy in the values is seen to decrease noticeably for single-tone sinusoidal inputs, because the quantization noise in this case tends to become more Gaussian. For $\Delta-\Sigma$ modulators whose inputs comprise of two sinusoids, the theoretical and simulated input stability limits are seen to be quite similar for relatively small input-amplitude signals. However, the difference increases as the amplitudes of the two sinusoids become larger. This is due to the occurrence of tones as the $\Delta-\Sigma$ modulator approaches its stability limit. A further reason for this discrepancy could be that the derivation of the three gains (i.e., two sinusoids and one Gaussian) is based on the modified nonlinearity concept. In order to compute the gain for any of the three inputs, it is assumed that the nonlinear function has been modified in turn by each of the two remaining inputs. However, in real-life, this may not be the case as all the three inputs coexist simultaneously.

\section{CONCLUSION}

The maximum stability input limits for different types of input signals and amplitudes were derived from the first principles and shown to be dependent on the quantizer gain as well as the stop-band attenuation of the NTFs. The derived stability curves were shown to depend on the noise-amplification factor,

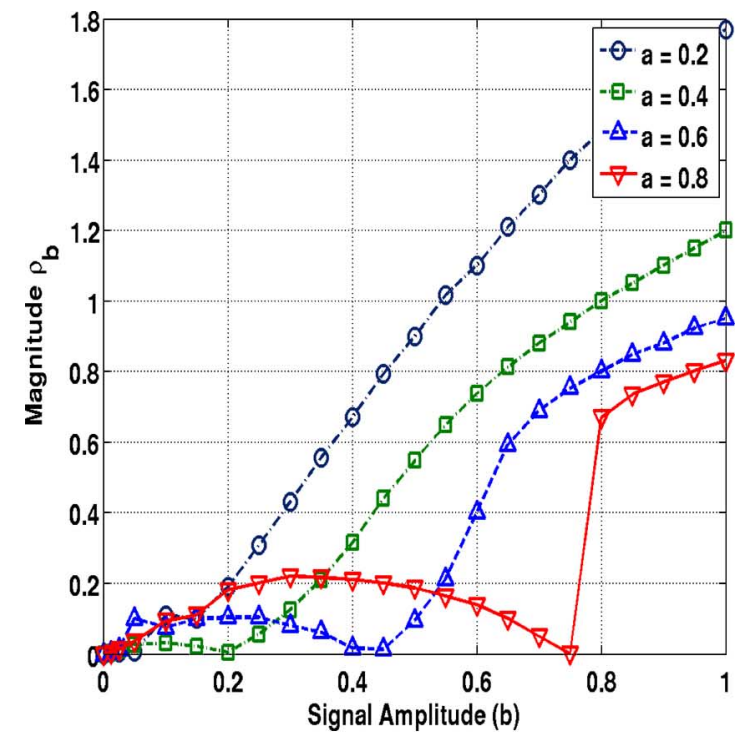

(a)

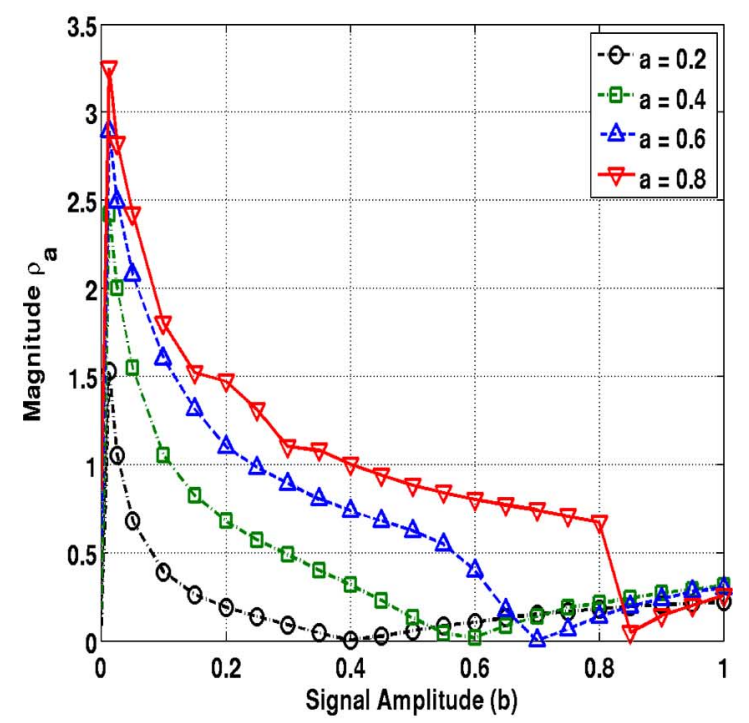

(b)

Fig. 13. (a) Variation of $\rho_{b}$ versus $b$ for different $a$ amplitudes. (b) Variation of $\rho_{a}$ versus $b$ for different $a$ amplitudes.

and therefore, the composition of the quantization noise of the $\Delta-\Sigma$ modulators. The theoretically derived stability curves were shown to agree reasonably well with the simulation results for various types of input signals and amplitudes. The stability limits for the sinusoidal-input signals were theoretically proved to be greater than the DC case for $\Delta-\Sigma$ modulators of the same order. This finding is particularly useful for the design of higher order $\Delta-\Sigma$ with improved SNRs and dynamic ranges. The derived stability curves will enable the designer of $\Delta-\Sigma$ modulators to predict with greater accuracy the stability of $\Delta-\Sigma$ modulators for any NTF and quantizer gain values.

\section{APPENDIX}

In this Appendix, the derivation of the gains for the two sinusoidal and Gaussian inputs to a 1-bit quantizer is made. 


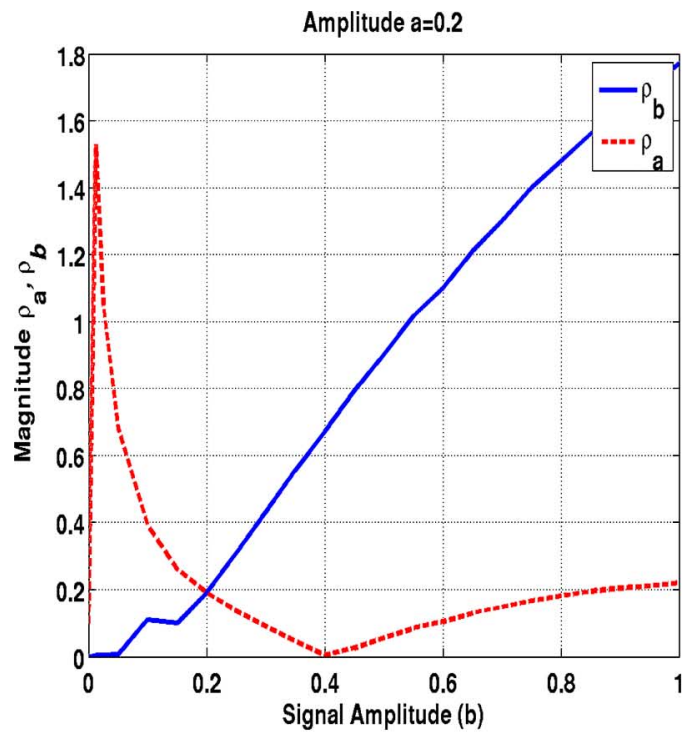

(a)

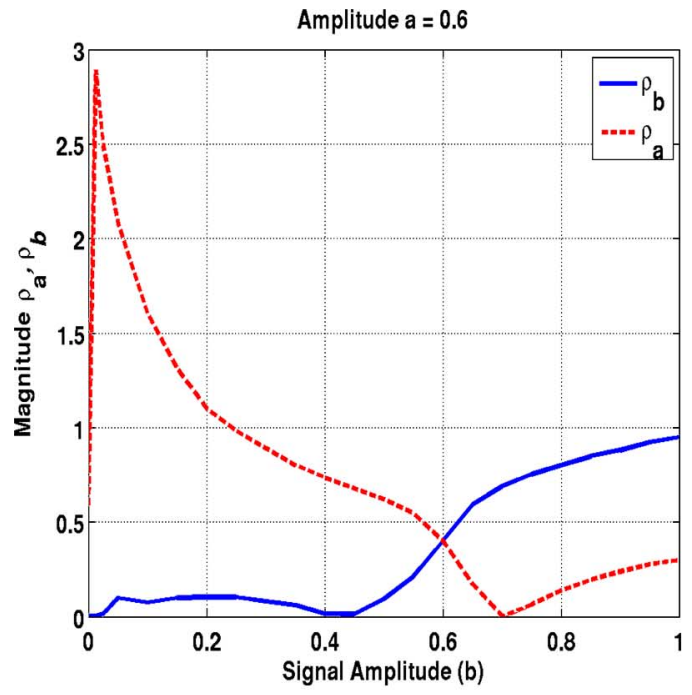

(c)

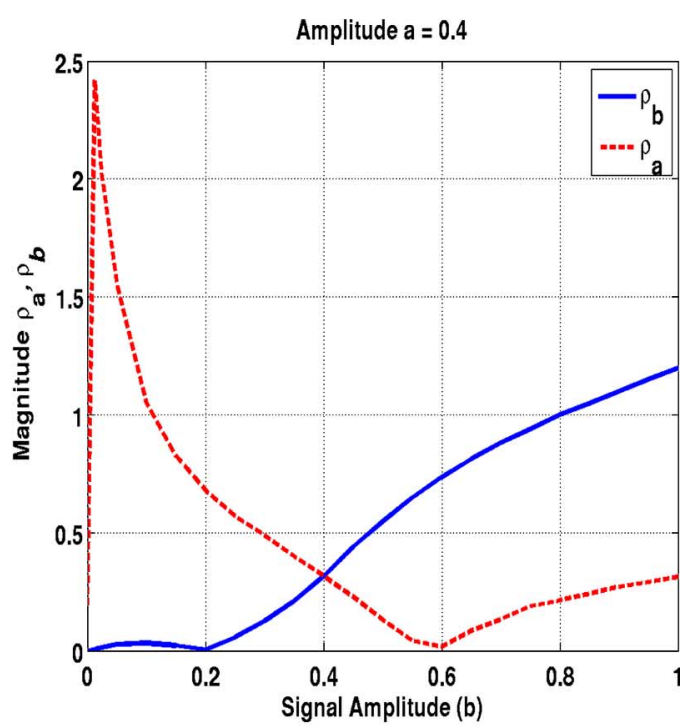

(b)

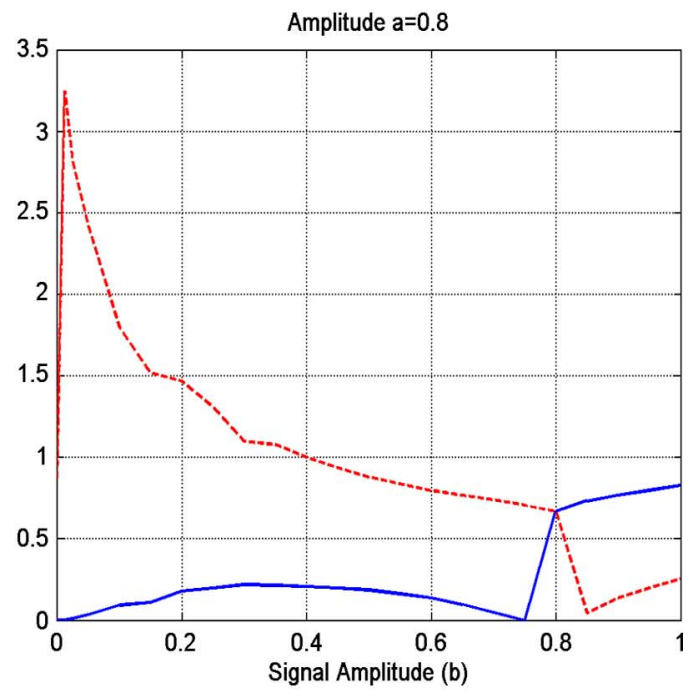

(d)

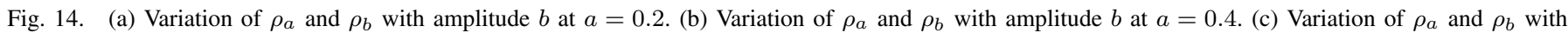
amplitude $b$ at $a=0.6$. (d) Variation of $\rho_{a}$ and $\rho_{b}$ with amplitude $b$ at $a=0.8$.

If the inputs to the nonlinearity are of different pdfs or of different magnitudes of similar waveforms, the output component from one of these inputs depends not only on the magnitude of this particular input but also on the magnitudes of all the other inputs. The concept used here is the modified-linearity concept [14], whereby to determine the response to a particular input, the nonlinear characteristic is modified in turn by each of the input signals present to obtain a modified nonlinearity to which the input is applied.

The two sinusoidal inputs considered here are $x_{a}(t)=$ $a \cos \left(w_{1} t+\phi_{1}\right)$ and $x_{b}(t)=b \cos \left(w_{2} t+\phi_{2}\right)$, where $a$ and $b$ are constants, $\omega_{1}$ and $\omega_{2}$ are the sinusoidal frequencies, assumed to be incommensurate, and $\phi_{1}$ and $\phi_{2}$ are RVs each having a uniform pdf in the interval $[0,2 \pi]$. The third input is the quantization noise assumed to be Gaussian $G(0, \sigma)$, i.e., with zero mean and variance $\sigma^{2}$.

\section{Sinusoidal Gains}

The modified nonlinearity of a 1-bit quantizer with a random input is given by [12]

$$
n_{1}(\gamma)=2 \Delta \int_{0}^{\gamma} q(y) d y
$$

where $\pm \Delta$ is the output of the 1-bit quantizer, and $q(y)$ is the pdf of the random input.

Therefore, for a Gaussian input

$$
n_{1}(\gamma)=2 \Delta \int_{0}^{\gamma}\left(\frac{1}{\sigma \sqrt{2 \pi}}\right) e^{\frac{-y^{2}}{2 \sigma^{2}}} d y
$$




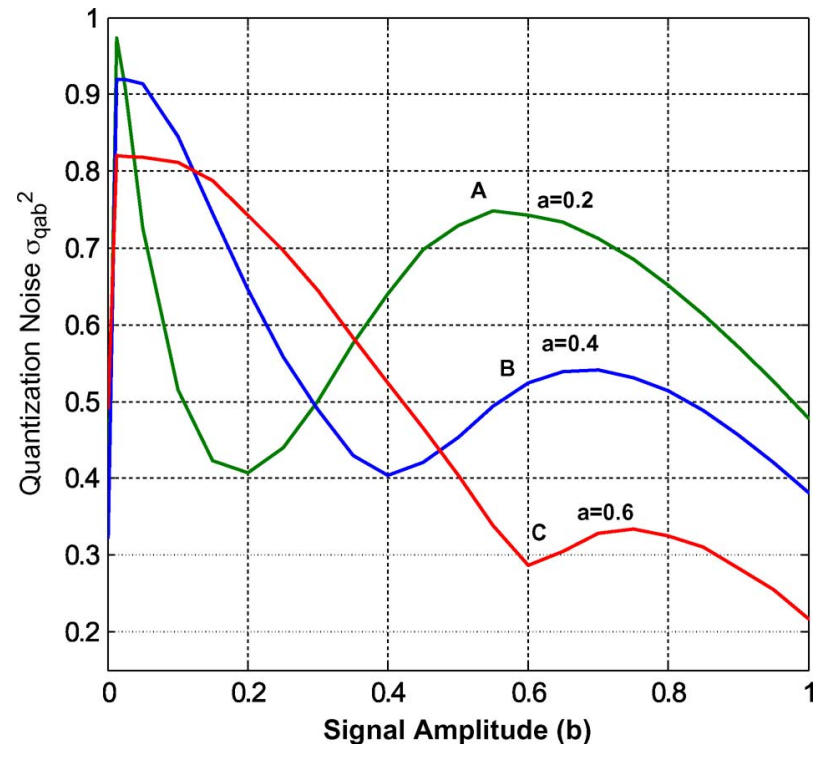

Fig. 15. Variation of quantization noise versus the two sine amplitudes.

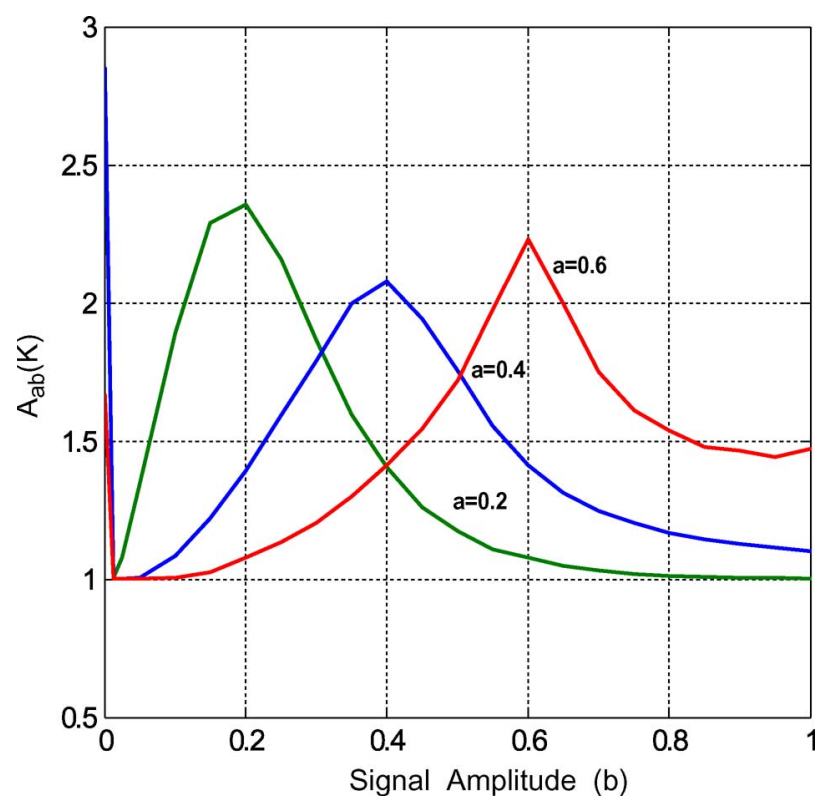

Fig. 16. $A_{a b}(k)$ variation versus the two sine amplitudes $a$ and $b$.

which, when integrated, simplifies to

$$
n_{1}(\gamma)=\Delta \operatorname{erf}\left(\frac{\gamma}{\sigma \sqrt{2}}\right)
$$

Next, we consider the nonlinearity $n_{1}(\gamma)$ that is further modified to $n_{2}(\gamma)$ by one of the sinusoidal signals, for example, $x_{a}(t)$. This further modified nonlinearity is given by [14]

$$
n_{2}(\gamma)=\int_{-a}^{a} p(x) n_{1}(x+\gamma) d x
$$

where $p(x)$ is the pdf of $x_{a}(t)$, i.e.,

$$
n_{2}(\gamma)=\int_{-a}^{a} \frac{1}{\pi} \frac{1}{\sqrt{a^{2}-x^{2}}} \Delta \operatorname{erf}\left(\frac{x+\gamma}{\sigma \sqrt{2}}\right) d x
$$

can be rewritten as

$$
n_{2}(\gamma)=\frac{2 \Delta}{\pi} \int_{0}^{a} \frac{1}{\sqrt{a^{2}-x^{2}}} \operatorname{erf}\left(\frac{x+\gamma}{\sigma \sqrt{2}}\right) d x .
$$

When integrating (A6), we get (A7), which is

$$
\begin{aligned}
n_{2}(\gamma)=\mid & {\left[e^{-\frac{(\gamma+x)^{2}}{2 \sigma^{2}}} \sigma \sqrt{\frac{2}{\pi}}+(\gamma+x) \operatorname{erf}\left(\frac{x+\gamma}{\sigma \sqrt{2}}\right)\right] } \\
& \left.-\left[\frac{\sigma^{2} x \operatorname{erf}\left(\frac{x+\gamma}{\sigma \sqrt{2}}\right)}{\left(a^{2}-x^{2}\right)}\right]\right\} \\
& \times\left.\left\{\frac{\left(a^{2}-x^{2}\right)^{\frac{3}{2}}}{\left(a^{2} x^{2}\right)\left(a^{2}+x \gamma\right)-\sigma^{2} a^{2}-2 x^{2} \sigma^{2}}\right\}\right|_{0} ^{a} .
\end{aligned}
$$

After applying the limits, (A7) simplifies to

$$
n_{2}(\gamma)=\left(\frac{2 \Delta}{\pi}\right)\left(\frac{a}{\sigma^{2}-a^{2}}\right)\left\{\sigma \sqrt{\frac{2}{\pi}} e^{\frac{-\gamma^{2}}{2 \sigma^{2}}}+\gamma \operatorname{erf}\left(\frac{\gamma}{\sigma \sqrt{2}}\right)\right\}
$$

where $n_{2}(\gamma)$ is now the nonlinearity of the 1-bit quantizer, which has been modified by the sinusoidal input $x_{a}(t)$ and the quantization noise $G(0, \sigma)$. The next step is to evaluate the gain for $x_{b}(t)$ to this modified nonlinearity. This gain for $x_{b}(t)$ would be a function of the input amplitudes $a$ and $b$ and would also depend on the quantization noise power $\sigma^{2}$.

The gain $K_{b}$ of the sinusoidal input $x_{b}(t)$ to this nonlinearity $n_{2}(\gamma)$ is given by [12]

$$
K_{b}=\frac{1}{\sigma_{b}^{2}} \int_{-b}^{b} x n_{2}(x) r(x) d x
$$

where $\sigma_{b}^{2}=b^{2} / 2$ is the variance, and $r(x)$ is the pdf of $x_{b}(t)$. From (A8) and (A9), we get the gain for $x_{b}(t)$ as in (A10), which is

$$
\begin{aligned}
K_{b}= & \left(\frac{2}{b^{2}}\right)\left(\frac{2 \Delta}{\pi^{2}}\right)\left(\frac{2 a}{\sigma^{2}-a^{2}}\right) \\
\times & {\left[\sigma \sqrt{\frac{2}{\pi}} \int_{0}^{b} e^{\frac{-x^{2}}{2 \sigma^{2}}} \frac{x}{\sqrt{b^{2}-x^{2}}} d x\right.} \\
& \left.+\int_{0}^{b} \frac{x^{2}}{\sqrt{b^{2}-x^{2}}} \operatorname{erf}\left(\frac{x}{\sigma \sqrt{2}}\right) d x\right] .
\end{aligned}
$$




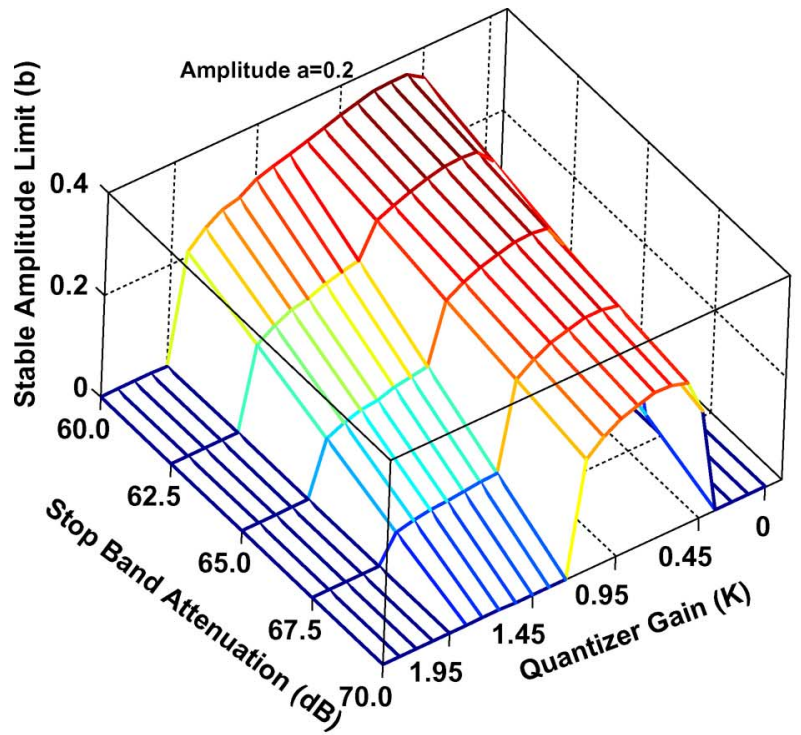

(a)

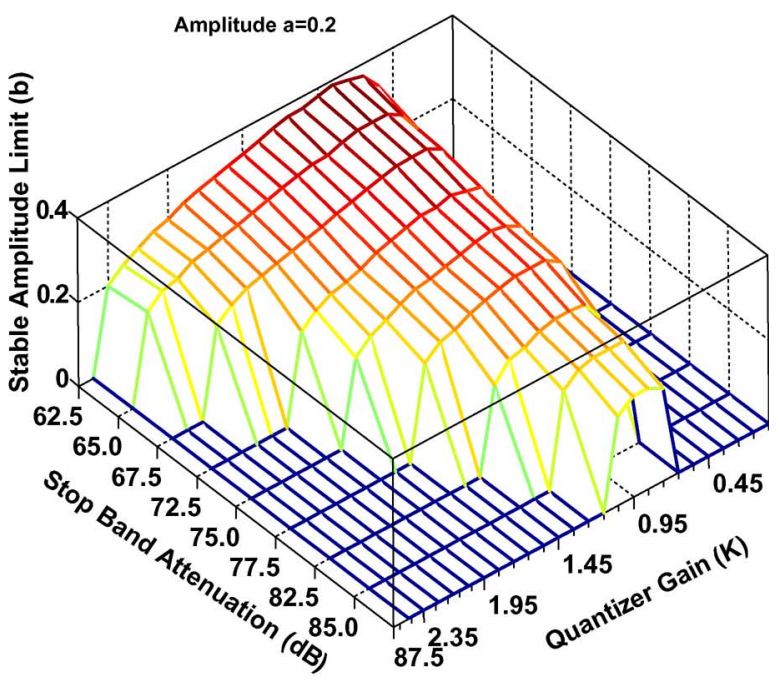

(b)

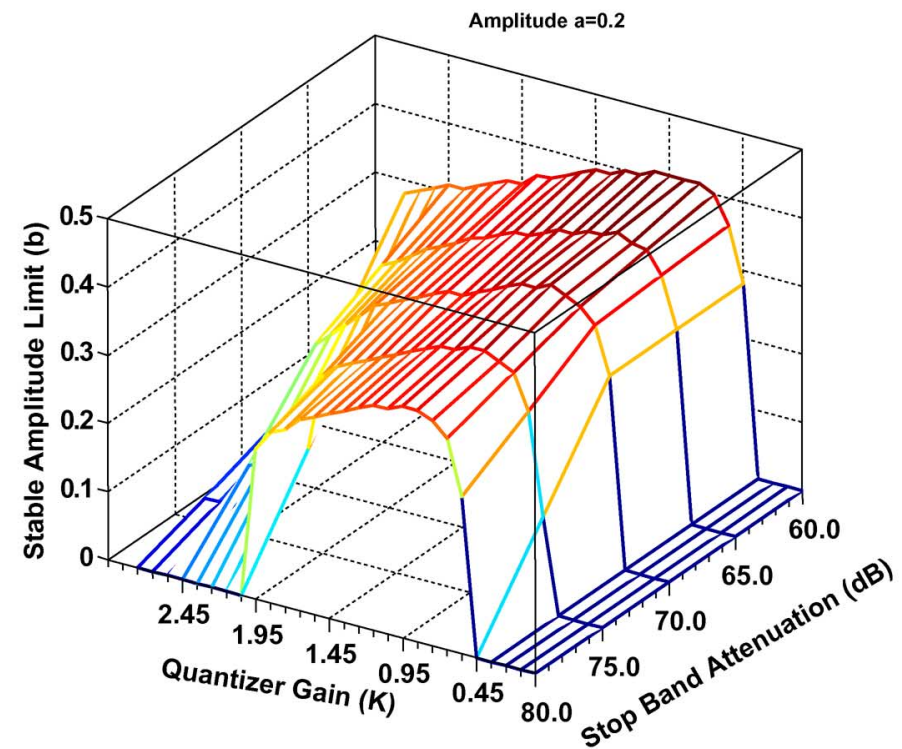

(c)

Fig. 17. (a) Stable input limits of amplitude $b$ of third order for $a=0.2$. (b) Stable input limits of amplitude $b$ of fourth order for $a=0.2$. (c) Stable input limits of amplitude $b$ of fifth order for $a=0.2$.

By putting $x=b u^{1 / 2}$, the first integral in (A10) can be simplified to

$$
I_{1}=\int_{0}^{b} e^{\frac{-x^{2}}{2 \sigma^{2}}} \frac{x}{\sqrt{b^{2}-x^{2}}} d x=\left(\frac{b}{2}\right) \int_{0}^{1} e^{-\rho_{b}^{2} u} u^{0}(1-u)^{\frac{1}{2}} d u
$$

where $\rho_{b}^{2}=b^{2} / 2 \sigma^{2}$. This reduces (A11) to the integral form of the confluent hypergeometric function ${ }_{1} F_{1}(\alpha, \beta, \lambda)$, which is [13]

$$
\frac{\Gamma(\beta)}{\Gamma(\alpha) \Gamma(\beta-\alpha)} \int_{0}^{1} e^{\lambda u} u^{\alpha-1}(1-u)^{\beta-\alpha-1} d u={ }_{1} F_{1}(\alpha, \beta, \lambda) .
$$

From (A11) and (A12), $I_{1}$ can be integrated as

$$
I_{1}=\left(\frac{b}{2}\right) \int_{0}^{1} e^{-\rho_{b}^{2} u} u^{0}(1-u)^{\frac{1}{2}} d u=b_{1} F_{1}\left(1, \frac{3}{2},-\rho_{b}^{2}\right) .
$$

The second integral in (A10) can be solved by expanding the error function and integrating within the limits, as shown in (A14)

$$
I_{2}=\int_{0}^{b} \operatorname{erf}\left(\frac{x}{\sigma \sqrt{2}}\right) \frac{x^{2}}{\sqrt{b^{2}-x^{2}}} d x=2 \frac{b^{2}}{\sqrt{\pi}} \eta
$$




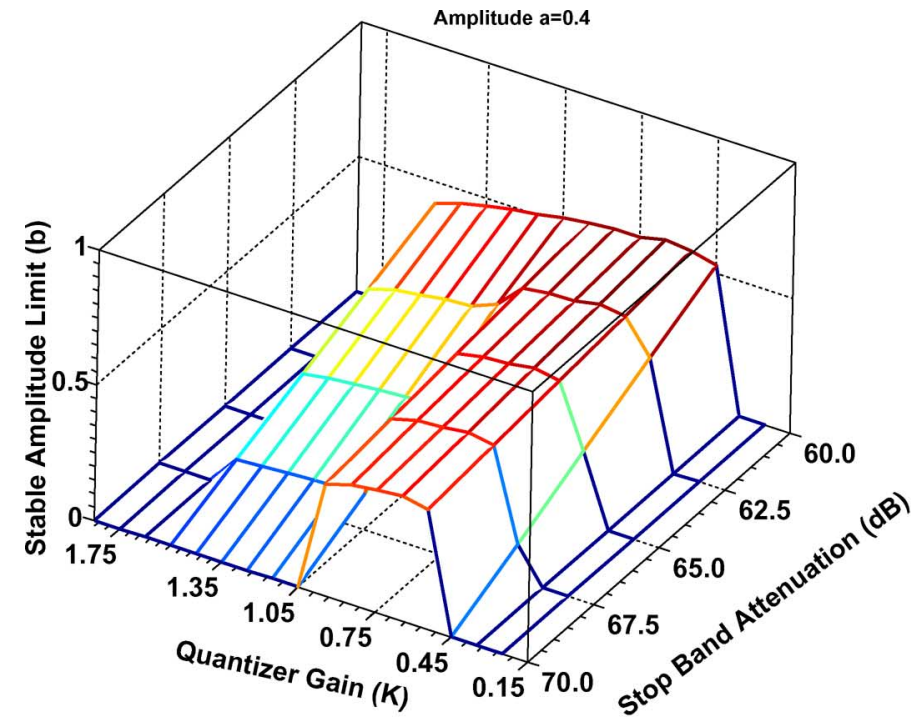

(a)

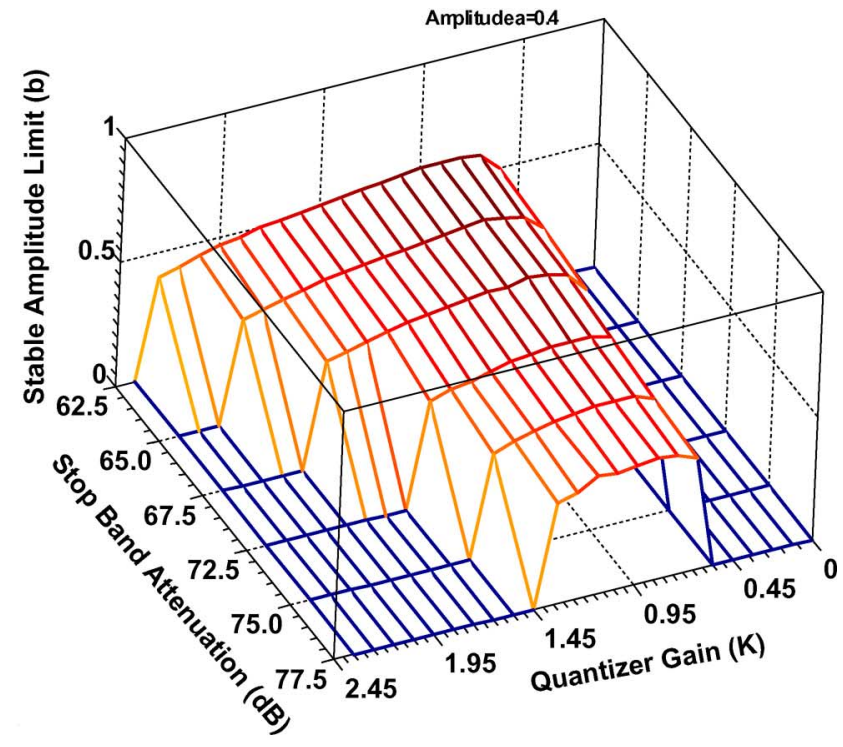

(b)

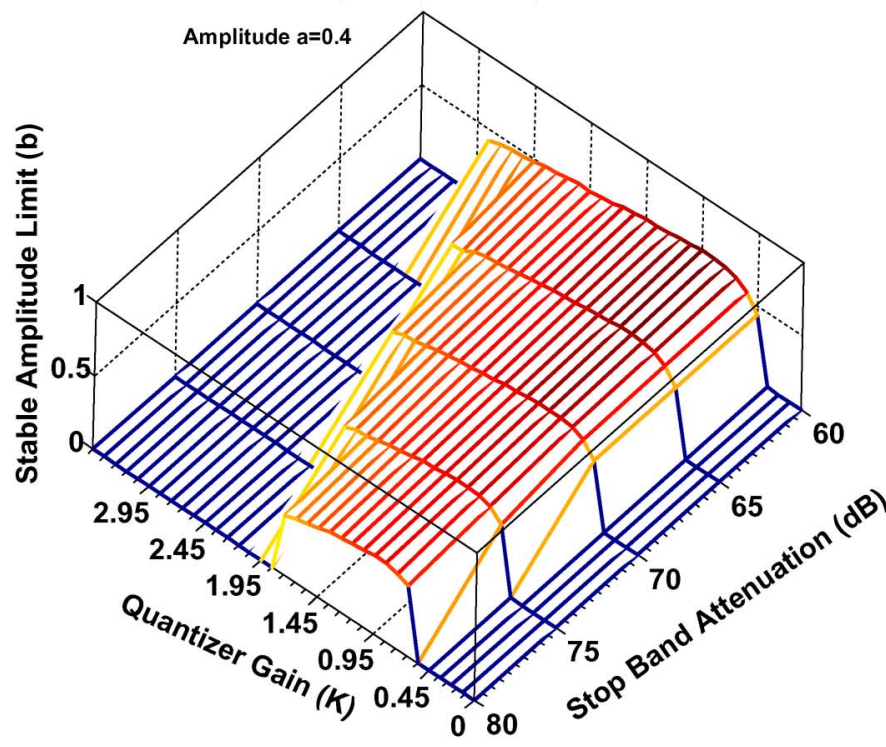

(c)

Fig. 18. (a) Stable input limits of amplitude $b$ of third order for $a=0.4$. (b) Stable input limits of amplitude $b$ of fourth order for $a=0.4$. (c) Stable input limits of amplitude $b$ of fifth order for $a=0.4$.

where $\eta$ is an infinite series given by

$$
\eta=\left\{\frac{2}{3} \rho_{b}-\frac{8}{45} \rho_{b}^{3}+\frac{8}{175} \rho_{b}^{5}-\frac{64}{6615} \rho_{b}^{7}+\ldots\right\} .
$$

From (A10), (A13), and (A14), we get

$$
\begin{aligned}
K_{b}=\left(\frac{2}{b^{2}}\right) & \left(\frac{2 \Delta}{\pi^{2}}\right)\left(\frac{2 a}{\sigma^{2}-a^{2}}\right) \\
& \times\left\{\sigma \sqrt{\frac{2}{\pi}} b_{1} F_{1}\left(1, \frac{3}{2},-\rho_{b}^{2}\right)+2 \frac{b^{2}}{\sqrt{\pi}}\right\} .
\end{aligned}
$$

Simplifying (A16) further and rearranging the terms, the gain $K_{b}$ for $x_{b}(t)$ is given by

$$
K_{b}=\left(\frac{2}{\pi}\right)^{\frac{5}{2}}\left(\frac{\Delta}{\sigma}\right)\left(\frac{a}{b}\right)\left(\frac{1}{\frac{1}{2}-\rho_{a}^{2}}\right)\left\{{ }_{1} F_{1}\left(1, \frac{3}{2},-\rho_{b}^{2}\right)+\psi_{b}\right\}
$$

where

$$
\psi_{b}=\left\{\frac{4}{3} \rho_{b}^{2}-\frac{16}{45} \rho_{b}^{4}+\frac{16}{175} \rho_{b}^{6}-\frac{128}{6615} \rho_{b}^{8}+\ldots\right\} .
$$




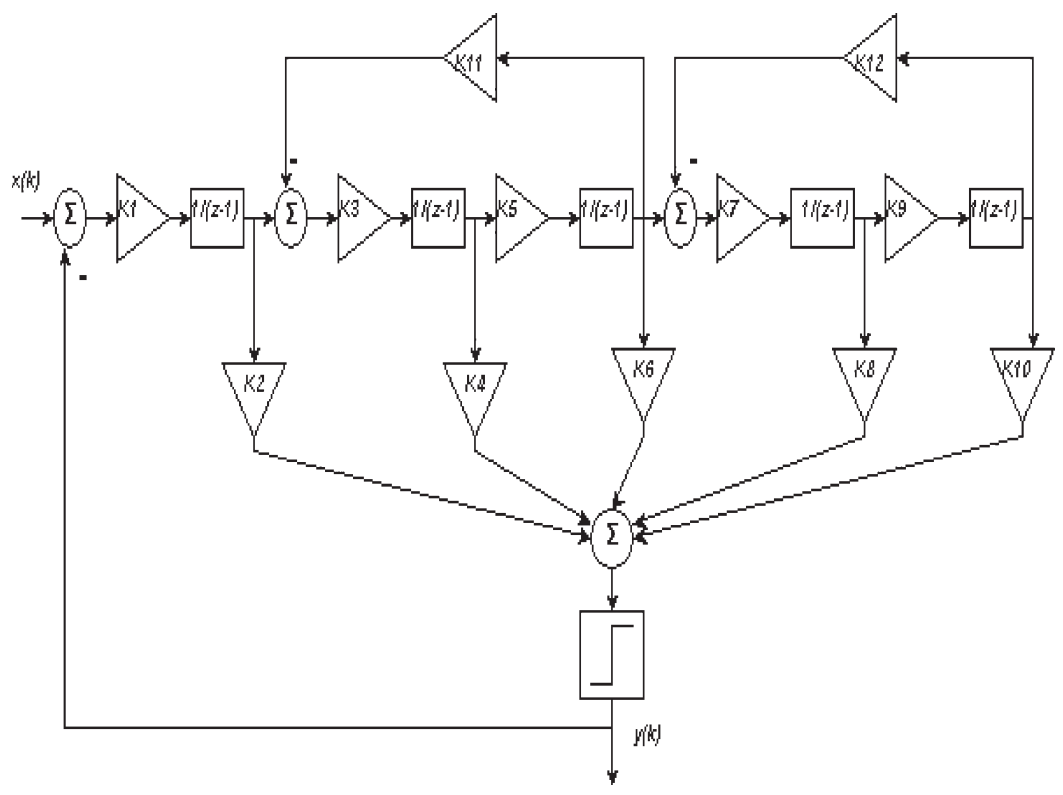

Fig. 19. Chebyshev-Type-II fifth-order modulator.

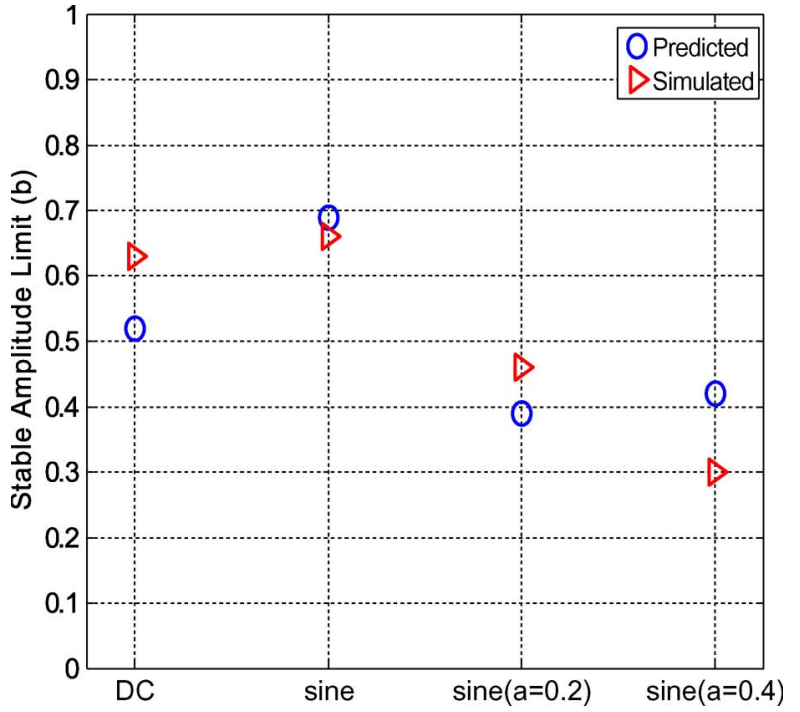

Fig. 20. Simulation results for dc, sine, and two sinusoidal inputs.

In order to obtain the gain for $x_{a}(t)$, we proceed as in above to get

$$
\begin{aligned}
K_{a}=\left(\frac{2}{\pi}\right)^{\frac{5}{2}}\left(\frac{\Delta}{\sigma}\right) & \left(\frac{b}{a}\right)\left(\frac{1}{\frac{1}{2}-\rho_{b}^{2}}\right) \\
& \times\left\{{ }_{1} F_{1}\left(1, \frac{3}{2},-\rho_{a}^{2}\right)+\psi_{a}\right\} .
\end{aligned}
$$

\section{Noise Gains}

The modified nonlinearity of the first order for a Gaussian input to a 1-b quantizer is given by [12]

$$
n(\sigma, \gamma)_{1}=\int_{-\infty}^{\infty} n(y+\gamma) H_{1}\left(\frac{y}{\sigma}\right) q(y) d y
$$

where $H_{1}$ is the Hermite polynomial of the first order. Substituting for $q(y)$ and $n(y+\gamma)$ in (A20)

$$
n(\sigma, \gamma)_{1}=\frac{\Delta}{\sigma^{2} \sqrt{2 \pi}} \int_{-\infty}^{\infty} y e^{-\frac{y^{2}}{2 \sigma^{2}}} d y=\sqrt{\frac{2}{\pi}} \Delta e^{-\frac{\gamma^{2}}{2 \sigma^{2}}}
$$

The noise gain $K_{n}$ in the presence of another random input with pdf $p(r)$ is given by [12]

$$
K_{n}=\frac{1}{\sigma} \int_{-\infty}^{\infty} n(\sigma, r){ }_{1} p(r) d r
$$

Here, we consider the additional random input as a combination of two uncorrelated sinusoidal inputs. The joint pdf $p(r)$ of the two sinusoidal signals having amplitudes $a$ and $b$, with incommensurate frequencies, is given by [15]

$$
p(r)=\frac{r}{\pi a b} \frac{1}{\sin \theta}
$$

where

$$
\theta=\cos ^{-1}\left(\frac{a^{2}+b^{2}-r^{2}}{2 a b}\right)
$$

From (A21), (A22), and (A23), we get

$$
K_{n}=\sqrt{\frac{2}{\pi}}\left(\frac{\Delta}{\sigma}\right) \int_{a-b}^{a+b} e^{-\frac{r^{2}}{2 \sigma^{2}}}\left(\frac{r}{\pi a b}\right)\left(\frac{1}{\sin \theta}\right) d r .
$$


Changing the variable from $r \rightarrow \theta$

$$
K_{n}=\sqrt{\frac{2}{\pi}}\left(\frac{\Delta}{\sigma \pi}\right) e^{-\frac{a^{2}}{2 \sigma^{2}}} e^{-\frac{b^{2}}{2 \sigma^{2}}} \int_{0}^{\pi} e^{k \cos \theta} d \theta
$$

where $k=a b / \sigma^{2}$. Solving the integral earlier, we get the noise gain as

$$
K_{n}=\sqrt{\frac{2}{\pi}}\left(\frac{\Delta}{\sigma}\right) e^{-\rho_{a}^{2}} e^{-\rho_{b}^{2} \zeta}
$$

where

$$
\zeta=\left\{1+\rho_{a}^{2} \rho_{b}^{2}+\frac{\rho_{a}^{4} \rho_{b}^{4}}{4}+\frac{\rho_{a}^{6} \rho_{b}^{6}}{36}+\frac{\rho_{a}^{8} \rho_{b}^{8}}{576}+\ldots\right\} .
$$

\section{REFERENCES}

[1] R. Schreier and W. M. Snelgrove, " $\Sigma \Delta$ modulation is a mapping," in Proc. ISCAS, 1991, vol. 5, pp. 2415-2418.

[2] S. Hien and A. Zakhor, "Stability and scaling of double loop $\Sigma \Delta$ modulators," in Proc. ISCAS, 1992, vol. 3, pp. 1312-1315.

[3] H. Wang, "A geometric view of $\Sigma \Delta$ modulation," IEEE Trans. Circuits Syst., vol. 39, no. 6, pp. 402-405, Jun. 1992.

[4] P. Steiner and W. Yang, "Stability analysis of the second-order $\Sigma \Delta$ modulator," in Proc. ISCAS, 1994, vol. 5, pp. 365-368.

[5] B. P. Agarwal and K. Shenoi, "Design methodology for $\Sigma \Delta$ modulators," IEEE Trans. Commun., vol. COM-31, no. 3, pp. 360-370, Mar. 1983.

[6] S. H. Ardalan and J. Paulos, "An analysis of nonlinear behavior in delta-sigma modulators," IEEE Trans. Circuits Syst., vol. CAS-34, no. 6, pp. 593-603, Jun. 1987.

[7] [Online]. Available: www.functions.wolfram.com

[8] K. C. H. Chao, S. Nadeem, W. L. Lee, and C. G. Sodini, "A higher order topology for interpolative modulators for oversampling A/D converters," IEEE Trans. Circuits Syst., vol. 37, no. 3, pp. 309-318, Mar. 1990.

[9] L. Risbo, "Stability predictions for high-order $\Sigma \Delta$ modulators based on quasilinear modeling," in Proc. IEEE Int. Symp. Circuits Syst., 1994, vol. 5, pp. 361-364.

[10] A. Gelb and W. E. Vander Velde, Multiple-Input Describing Functions and Nonlinear System Design. New York: McGraw-Hill, 1968.

[11] E. F. Stikvoort, "Some remarks on the stability and performance of the noise shaper or sigma-delta modulator," IEEE Trans. Commun., vol. 36, no. 10, pp. 1157-1162, Oct. 1988.

[12] D. P. Atherton, Nonlinear Control Engineering: Describing Function Analysis and Design. London, U.K.: Van Nostrand Reinhold, 1982, pp. 383-388.

[13] A. H. Haddad, "Nonlinear systems," in Benchmark Papers in Electrical Engineering and Computer Science, vol. 10. Dowden, Hutchinson \& Ross, Inc., 1975, p. 197.

[14] D. P. Atherton and G. F. Turnbull, "Response of nonlinear characteristics to several inputs and the use of the modified linearity concept in control systems," Proc. Inst. Electr. Eng., vol. 111, no. 1, pp. 157-164, Jan. 1964.

[15] A. K. Mahalanabis and A. K. Nath, "On the dual-input describing functions of a nonlinear element," IEEE Trans. Autom. Control, vol. AC-10, no. 2, pp. 203-204, Apr. 1965.

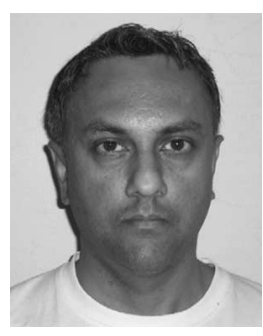

Jaswinder Lota (M'01) received the B.Sc. degree from the National Defence Academy, Pune, India, in 1987, the B.Eng. degree in electrical engineering from the Naval College of Engineering, Lonavla, India, in 1991, the M.Eng. degree in radar and communication engineering from the Indian Institute of Technology, New Delhi, India, in 1997, and the $\mathrm{Ph} . \mathrm{D}$. degree from the University of Westminster, London, U.K., in 2007.

From 1989 to 2004, he was with the Indian Navy and was last appointed as Deputy Director Systems with the Naval Headquarters, New Delhi. From 2004 to 2006, he was a research scholar with the University of Westminster. Since 2006, he has been with Sepura plc., Cambridge, U.K., as a Senior Engineer, working on developing new technologies, algorithms, standards, and techniques relevant to the future product development for TETRA-2 radio equipment. His research interests include sigma-delta modulators, digital-signal processing, radar, sonar, and telecommunication systems.

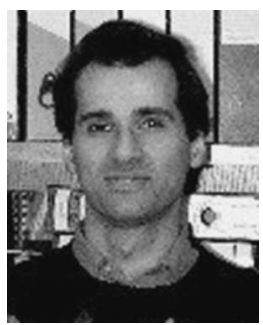

Mohammed Al-Janabi (S'98-A'00-M'04) received the B.Eng. degree (with honors) in electronic engineering and the Ph.D. degree in digital-signal processing from the University of Westminster, London, U.K., in 1993 and 2000, respectively.

He has been a Senior Lecturer with the Applied DSP and VLSI Research Group, Department of Electronics, Communications, and Software Engineering, University of Westminster, since 2000. He teaches analog and digital circuit design. He also supervises projects for M.Sc. and Ph.D. degree students. His research interests include digital-signal processing, data converters, sigma-delta modulation, and control theory.

Dr. Al-Janabi is a chartered member of the Institute of Engineering and Technology (formerly IEE).

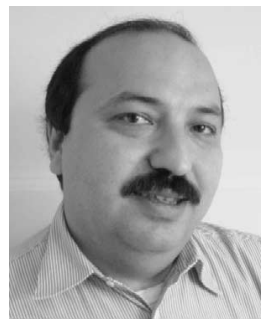

Izzet Kale (M'88) was born in Cyprus. He received the B.Sc. degree (with honors) in electrical and electronic engineering from the Polytechnic of Central London, London, U.K., the M.Sc. degree in design and manufacture of microelectronic systems from Edinburgh University, Edinburgh, U.K., and the Ph.D. degree in techniques for reducing digital filter complexity from the University of Westminster, London.

Since 1984, he has been with the staff of the University of Westminster (formerly, the Polytechnic of Central London), where he is currently a Professor of applied DSP and very large scale integration (VLSI) Systems, the Founder and Director of the Applied DSP and VLSI Research Group, where he has undertaken numerous applied research and development projects and contracts for European, U.S., and Japanese corporations working on innovative silicon-integrated product development for commercial applications. His research and teaching activities include digital- and analog-signal processing, silicon circuit and system design, digital-filter design and implementation, and A/D and D/A sigma-delta converters. He is currently working on efficiently implementable ultralowpower DSP algorithms/architectures and sigma-delta modulator structures for use in the communications and biomedical industries as well as hybrid Global Navigation Satellite Systems GPS/Galileo receiver structures and systems. 